\title{
Quantitative Scale for Grading of Cardiovascular Autonomic Reflex Tests and Small Fibers from Skin Biopsies (QASAT)
}

\section{Peter Novak*}

Department of Neurology, University of Massachusetts Medical School, University Campus, S4-810D, 55 Lake Avenue North Worcester, MA 01655, USA

\begin{abstract}
Background: Orthostatic intolerance including dizziness and syncope is common and may reflect autonomic dysfunction. Cardiovascular reflex tests (deep breathing, Valsalva maneuver and tilt test) are established diagnostic methods for evaluation of orthostatic and autonomic symptoms. Small fiber neuropathy, a frequent underlying mechanism, is evaluated by the quantitative sudomotor axonal reflex test (QSART) and skin biopsies. A comprehensive quantitative scale to grade abnormalities in these tests is lacking.

Methods: This study defines the QASAT - Quantitative scale for grading of cardiovascular reflex tests using heart rate, blood pressure, Transcranial Doppler, QSART and small fibers (intrapidermal sensory and sweat gland) densities from skin biopsies. The QASAT has three main categories: cardiovascular, cerebral blood flow (includes cerebral autoregulation/vasoreactivity score) and small fiber neuropathy. QASAT was validated in 612 participants with diabetes mellitus (92), Parkinson's disease (88), multiple system atrophy (23) and other diagnoses (409). The QASAT was compared with the Composite Autonomic Severity Score using ANOVA, correlations and sensitivity/specificity analysis.

Results: Scores of heart rate variability from deep breathing, orthostatic hypotension, orthostatic cerebral blood flow, sensory and sweat gland small fiber densities were disease specific $(p<0.0001)$ and were correlated with the severity of autonomic failure $(r=0.84, p<0.0001)$. Autonomic, sensory and cerebral blood flow abnormalities coexist and are correlated. Initial response to slow and fast tilt, baroreflex gain, QT interval, catecholamines and spectral analysis of the heart rate failed to correlate with severity of autonomic failure and therefore were not included in QASAT.

Conclusion: QASAT is an objective and validated instrument for grading of dysautonomia, associated small fiber neuropathy and cerebral blood flow. QASAT quantifies both below (bradycardia, supine/orthostatic hypotension) and above (tachycardia, supine/orthostatic hypertension) normal values. Cardiovascular tests are enhanced by scoring the cerebral hypoperfusion which underlies orthostatic symptoms. Inclusion of epidermal and sweat gland fiber density scoring improves lesion localization.
\end{abstract}

Keywords: QASAT; Autonomic; Small fiber neuropathy; Heart rate variability; Skin biopsy; Intraepidermal fibers; Sweat gland fibers

\section{Introduction}

Symptoms of orthostatic intolerance including syncope, dizziness and unexplained falls affect millions people worldwide, and may result in long-term disability. Syncope affects $35-45 \%$ of population [1-3], accounts for $1-3 \%$ of emergency visits and up to $6 \%$ of all hospitalizations [4]. The majority of syncope cases remain unexplained (37-50\%) $[5,6]$ although the known causes of syncope include reflex syncope $(21 \%)$, orthostatic hypotension (9.4\%) and cardiac (9.5\%) [5]. Dizziness affects $30 \%$ of elderly [7], and orthostatic dizziness accounts for $12.5 \%$ [8] and in $39 \%$ of patients no specific diagnosis can be found [9].

Cardiovascular reflex tests that include tilt test, deep breathing and Valsalva maneuver are clinically established tests to evaluate patients with orthostatic intolerance [10-12]. The tilt test can detect syncope, orthostatic hypotension and cerebral hypoperfusion that underlie orthostatic symptoms. Mechanisms provoked by the tilt are complex and include combinations of autonomic, humoral and cardiovascular responses [13-15]. Valsalva maneuver and deep breathing test are used to further refine the integrity of cardiovascular reflex circuits. Valsalva maneuver assesses predominantly adrenergic functions [16] while deep breathing test evaluates mainly parasympathetic functions [17]. Small fiber autonomic and sensory neuropathy is a common underlying mechanism of orthostatic intolerance [18-23], and can be further evaluated using the quantitative sudomotor axonal reflex test (QSART) [10-12] and skin punch biopsies for assessment of small fibers [24-28].

The above tests are sensitive to differentiate between normal and abnormal findings, but have limited ability to track the disease progression and severity. For example orthostatic hypotension can range from mild, transient, causing only minimal discomfort. On the other part of spectrum is severe orthostatic hypotension associated with a progressive drop of the blood pressure and cerebral blood flow upon standing causing syncope and falls. Both syncope and falls affect quality of life and pose a high risk for long-term disability [29-34].

Composite Autonomic Severity Score (CASS) is used for grading of autonomic failure using cardiovascular reflex tests and QSART in three domains: cardiovagal, adrenergic and sudomotor [35]. CASS has several limitations. Cardiovagal domain that is derived from the deep breathing test does not account for baseline heart rate and other mechanisms underlying changes in heart rate variability. Adrenergic failure is defined as a combination of abnormal blood pressure responses to Valsalva maneuver and the presence of orthostatic hypotension, but common findings such as supine hypo-/hypertension or excessive heart

*Corresponding author: Peter Novak MD, Department of Neurology, University of Massachusetts Medical School, University Campus, S4-810D, 55 Lake Avenue North Worcester, MA 01655, USA, Tel: 508-334-2527; Fax: 508-856-6778; E-mail: novakp@ummhc.org

Received February 05, 2015; Accepted April 15, 2015; Published April 17, 2015

Citation: Novak P (2015) Quantitative Scale for Grading of Cardiovascular Autonomic Reflex Tests and Small Fibers from Skin Biopsies (QASAT). J Neurol Disord 3: 226. doi:10.4172/2329-6895.1000226

Copyright: (c) 2015 Novak P. This is an open-access article distributed under the terms of the Creative Commons Attribution License, which permits unrestricted use, distribution, and reproduction in any medium, provided the original author and source are credited. 
rate increment to tilt are not quantified. Furthermore, CASS does not grade severity of small fiber neuropathy using skin biopsies but relies on QSART in grading postganglionic sudomotor deficit.

The new scale (Quantitative Autonomic Reflex and Small Fibers Tests - (QASAT)) is proposed. QASAT grades cardiovascular reflex tests, QSART and small fiber densities obtained from skin biopsies. QASAT was validated in 612 patients with a variety of disorders using training and a testing datasets. The sensitivity and specificity has been compared with CASS.

\section{Material and Methods}

\section{Ethics statement}

This study used data from standardized clinical tests. No modifications of tests were done to accommodate for this study. The study was approved by the Institutional Review Board of the University of Massachusetts Medical School as a minimal risk study, therefore the consent was waived.

\section{Study participants}

The study participants were consecutive patients referred to the Autonomic Laboratory at the Department of Neurology, University of Massachusetts Medical School between 2008-2013. In general, patients were referred for evaluation of autonomic failure, orthostatic hypotension or autonomic symptoms; or for evaluation of small fiber neuropathy or their combination. Most common autonomic symptoms were orthostatic lightheadedness, sudomotor complaints, and sphincter problems. Most common symptoms indicative of small fiber sensory neuropathy were distal pain/numbness/tingling. All patients were tested using the same laboratory protocol. Details about the inclusion and exclusion criteria have been published elsewhere [16].

\section{Cardiovascular reflex and sensory small fiber testing}

Our testing protocol used established standards [10,35,36] and was previously described [36]. Cardiovascular tests included deep breathing, Valsalva maneuver and tilt test. Sudomotor functions were assessed by the QSART. Signals were recorded using LabChart 7 system (ADInstruments, Inc. Colorado Springs, CO) and sampled at $400 \mathrm{~Hz}$ and included electrocardiogram (ECG), blood pressure, respiratory movement using a nasal thermistor, end tidal $\mathrm{CO}_{2}$ and blood flow velocity in the middle cerebral artery using Transcranial Doppler. Deep breathing test was performed at the rate of 6 breaths per minute for 1 minute. Valsalva maneuver was performed in the supine position with an expiratory pressure equal to $40 \mathrm{~mm} \mathrm{Hg}$ for 15 seconds. Participants were allowed to rest for 10 minutes in supine position and were then tilted to the upright position at 70 degrees for at least 10 minutes if tolerated. QSART was performed using the Q-Sweat machine (WR Medical Electronics, Stillwater, $\mathrm{MN}$ ) at regional limb temperature above $29.9^{\circ} \mathrm{C}$ with the simulation current $2 \mathrm{~mA}$ for 5 minutes, and the sweat volume was collected for 10 minutes. Baseline supine blood pressure was obtained intermittently using an automated blood pressure device Dinamap ProCare Monitor 100 (GE, Fairfield, CT) and continuously (beat-to-beat) using Finometer ${ }^{\oplus}$ (Finapress Medical Systems, Amsterdam, Netherlands). During the tilt test, the blood pressure was obtained every minute using the Dinamap ProCare and continuously using Finometer ${ }^{\oplus}$. Normative data for deep breathing test, blood pressure response to the Valsalva maneuver and tilt test, adjusted for age and sex if appropriate, were published previously $[10,27,28,36]$. The temporal acoustic window with a $2 \mathrm{MHz}$ Transcranial Doppler probe was used for acquisition of blood flow velocity signals using a MultiDop T (DWL, Singen, Germany). Normal values are age- and sex- dependent. Our limits of normality $\left(5^{\text {th }}\right.$ percentile $)$ and are defined by the linear equations:
(1) $\mathrm{Men}_{\text {lower limit }}=72.09-0.38 *$ age;
(2) $\mathrm{Men}_{\text {upper limit }}=84.35-0.38 *$ age;
(3) Women $_{\text {lower limit }}=82.2-0.45^{\star}$ age;
(4) Women $_{\text {upper limit }}=90.8-0.45^{*}$ age;

For the Valsalva maneuver, normal response is defined as systolic blood flow velocity $\geq 85 \%$ of the baseline at the end of the phase 2 .

Epidermal sensory fiber density (ENFD) and sweat gland fiber density (SGFD) were obtained using 3-mm full-thickness skin punch biopsies taken from the lateral thigh $(20 \mathrm{~cm}$ distal to the iliac spine) and distal leg ( $10 \mathrm{~cm}$ above the lateral malleolus) and stained with PGP 9.5 antibody [23-26]. The samples were processed at Therapath (New York, NY). The normal values for both ENFD and SGFD were determined by Therapath. The limits of normality for ENFD ( $5^{\text {th }}$ percentile) at the thigh were 6.2/8.3 (men/women) fibers per millimeter of epidermal length. Our limits of normality for ENFD for the distal leg are:

(5) $\mathrm{Men}_{\text {low limit }}=9.5-0.075^{\star}$ age;

(6) Women $_{\text {low limit }}=11.1-0.08^{\star}$ age;

The limits of normality for SGFD at the thigh and distal leg were 37.8 and 36.5 fibers per millimeter.

\section{Validation of other tests}

Additional tests that were also tried include fast tilt test, baroreflex gain, Q-T interval, spectral analysis of heart rate, catecholamines and cerebral autoregulation. All these tests were implemented as recommended. Initial heart rate and blood pressure response to tilt depend on the adrenergic function [37] and may be used instead of the Valsalva maneuver, which requires demanding straining that can be difficult to achieve in frail patients. The baseline, minimum, and maximum BP were obtained during first 30 seconds of the tilt. The test was performed twice, both times at 70 degrees, once with slow tilt (7 seconds) and once with fast tilt ( 3 seconds). The order of the tilts was random, and there was a pause of at least 20-minute between the two tilts. Baroreflex gain [19-21,38] and QT interval [38] which are prognostic markers of dysautonomia, were also tested. Baroreflex gain was calculated as described by Bernardi et al. [38] The QT interval corrected for heart rate using Bazett's formula was obtained via the ECG module of the LabChart software.

Low frequency (LF) and high frequency (HF) oscillations in RR intervals are thought to reflect sympathetic and parasympathetic functions, respectively [39,40]. LF and HF, both absolute and normalized, and their ratios were calculated in the frequency domain according to recommended standards $[11,40]$ using the Heart Rate Variability module of LabChart. Plasma catecholamines which are indices of activity of the sympathetic nervous system [41,42] were also evaluated. Blood samples for norepinephrine testing (Quest Diagnostics, Chantilly, VA,) were obtained from patients in the supine position and again after 3 minutes of standing. Plasma metanephrines and normetanephrines (Quest Diagnostics, Chantilly, VA)) were obtained in the supine position only.

Cerebral autoregulation is a term for complex mechanisms that determines the pressure-flow relationship. Its assessment can be useful in evaluation of patients with syndromes associated with cerebral hypoperfusion $[43,44]$. Several algorithms were tried to determine 
Citation: Novak P (2015) Quantitative Scale for Grading of Cardiovascular Autonomic Reflex Tests and Small Fibers from Skin Biopsies (QASAT). J Neurol Disord 3: 226. doi:10.4172/2329-6895.1000226

Page 3 of 11

the most optimal index of cerebral autoregulation including linear regression as a marker of static autoregulation [44] and transfer function analysis as a marker of dynamic autoregulation [45]. In addition, methods proposed by Tiecks et al. [46,47] were also explored using Valsalva maneuver for assessment of dynamic autoregulation and tilt test for assessment of static autoregulation.

\section{QASAT}

The QASAT (Table 1) is based on CASS [35]. CASS provides a quantitative estimation of autonomic failure in cardiovagal (score $0-3$ ), adrenergic (score 0-4), and sudomotor (score 0-3) domains as well as total score (0-10). CASS stratifies autonomic failure as none (total score 0 ), mild (total score 1-3), moderate (total score 4-6), or severe (total score 7-10).

\section{Heart rate}

Cardiovascular

\begin{tabular}{|l|l|l|l|}
\hline 1.Bradycardia - Supine and/or during tilt & \multicolumn{2}{l|}{} \\
\hline Results & Grading & Value & Definition \\
\hline Normal & & 0 & $H R \geq 50$ BPM \\
\hline Abnormal & & 1 & HR $<50$ BPM \\
\hline
\end{tabular}

${ }^{*} \mathrm{HR}=$ heart rate, $\mathrm{BPM}=$ beats per minute, at least 10 minutes of the rest should be obtained

2.Tachycardia - Supine

\begin{tabular}{|l|l|l|l|}
\hline Results & Grading & Value & Definition \\
\hline Normal & & 0 & HR $\leq 100$ BPM \\
\hline Abnormal & Mild & 1 & HR $>100$ BPM \\
\hline
\end{tabular}

$1 \quad H R>100 B P M$

3.Increased heart rate response to tilt

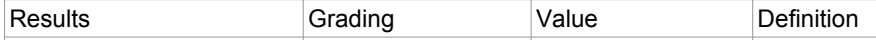

Normal

\begin{tabular}{|c|c|c|c|}
\hline Normal & & 0 & $\mathrm{HR}$ increment $\geq 10 \mathrm{BPM}$ and $<30 \mathrm{BPM}$ during tilt and $\mathrm{HR} \leq \mathbf{1 1 0}$ during tilt \\
\hline \multirow{3}{*}{ Abnormal } & Mild & 1 & $\begin{array}{l}\mathrm{HR}>110 \text { but } \leq 120 \text { BPM during tilt only or } \\
\mathrm{HR} \text { increment during tilt }>30 \mathrm{BPM} \text { and } \mathrm{HR} \leq 120 \mathrm{BPM} \text { during tilt }\end{array}$ \\
\hline & Moderate & 2 & $\begin{array}{l}\mathrm{HR}>120 \mathrm{BPM} \text { and }<150 \mathrm{BPM} \text { and } \mathrm{HR} \text { increment during tilt }>30 \mathrm{BPM} \text { or } \\
\mathrm{HR}>150 \mathrm{BPM} \text { and } \mathrm{HR} \text { increment during tilt }<30 \mathrm{BPM}\end{array}$ \\
\hline & Severe & 3 & $\mathrm{HR}>150 \mathrm{BPM}$ and $\mathrm{HR}$ increment during tilt $>30 \mathrm{BPM}$ \\
\hline \multicolumn{4}{|c|}{ At least 10 minutes of tilt should be obtained } \\
\hline \multicolumn{4}{|c|}{ 4.Reduced heart rate response to tilt } \\
\hline Results & Grading & Value & Definition \\
\hline Normal & & 0 & HR increment $\geq 10$ BPM and $<30$ BPM during tilt \\
\hline Abnormal & & 1 & HR increment during tilt $<10 \mathrm{BPM}$ \\
\hline \multicolumn{4}{|c|}{ 5. Heart rate variability } \\
\hline Results & Grading & Value & Definition \\
\hline Normal & & 0 & *Heart rate variations to deep breathing test \\
\hline \multirow{2}{*}{ Abnormal } & Mild & 1 & abnormal but $\geq 50 \%$ of normal value ${ }^{*}$ \\
\hline & Moderate & 2 & $<50 \%$ but $\geq 35 \%$ of normal value \\
\hline \multicolumn{4}{|c|}{ Blood pressure } \\
\hline \multicolumn{4}{|c|}{ 6.Supine hypotension } \\
\hline Results & Grading & Value & Definition \\
\hline Normal & Normal & 0 & \\
\hline Abnormal & Abnormal & 1 & $\mathrm{SBP}<90$ or $\mathrm{DBP}<60$ \\
\hline \multicolumn{4}{|c|}{$\mathrm{SBP}=$ systolic blood pressure, $\mathrm{DBP}=$ diastolic blood pressure } \\
\hline \multicolumn{4}{|c|}{ 7.Orthostatic hypotension during tilt } \\
\hline Results & Grading & Value & Definition* \\
\hline Normal & & 0 & \\
\hline \multirow{4}{*}{ Abnormal } & Mild & 1,2 & \\
\hline & Moderate & 3,4 & \\
\hline & Severe & 5,6 & \\
\hline & Marked & $>6$ & \\
\hline
\end{tabular}

BP should be obtained at baseline, and every minute of tilt up 10 minutes of the tilt. For tilt longer than 10 minutes, additional measurements should be obtained every 5 minutes. At each measurement, assign the value 0 for normal results and 1 for abnormal results. *Abnormal result is defined as a systolic BP $\leq 80 \%$ of baseline or a mean BP $\leq 85 \%$ of baseline. Baseline is defined as the BP in supine position just before the tilt test.

\section{Valsava maneuver-blood pressure}

\begin{tabular}{|l|l|l|l|}
\hline Results & Grading & Value & Definition* $^{*}$ \\
\hline Normal & & 0 & End of phase 2 returns to or exceeds the baseline
\end{tabular}

\section{End of phase 2 fails to return to baseline, the reduction is:}

\begin{tabular}{l|l|l|l}
\hline Abnormal & Mild & 1 & $\geq 90 \%$ of baseline
\end{tabular}

\begin{tabular}{|l|l|l|l|}
\hline Abnormal & 2 & $<90 \%$ but $\geq 60 \%$ of baseline \\
\hline Moderate & 3 & $<60 \%$ of baseline
\end{tabular}

*Use mean BP expressed as changes in percent. Baseline is defined as the last mean BP just before Valsalva maneuver. The flat top response defined by minimum of phase 2 above the baseline is discarded. 
Citation: Novak P (2015) Quantitative Scale for Grading of Cardiovascular Autonomic Reflex Tests and Small Fibers from Skin Biopsies (QASAT). J Neurol Disord 3: 226. doi:10.4172/2329-6895.1000226

Page 4 of 11

\begin{tabular}{|c|c|c|c|}
\hline \multicolumn{4}{|c|}{ 9.Supine hypertension } \\
\hline Results & Grading & Value & Definition* \\
\hline Normal & & 0 & $\mathrm{SBP}<140$ or $\mathrm{DBP}<90$ \\
\hline \multirow{3}{*}{ Abnormal } & Mild & 1 & $(\mathrm{SBP} \geq 140$ or $\mathrm{DBP} \geq 90)$ and $(\mathrm{SBP}<160$ or $\mathrm{DBP}<100)$ \\
\hline & Moderate & 2 & $(\mathrm{SBP} \geq 160$ or $\mathrm{DBP} \geq 100)$ and $(\mathrm{SBP}<180$ or $\mathrm{DBP}<110$ \\
\hline & Severe & 3 & $\mathrm{SBP} \geq 180$ or $\mathrm{DBP} \geq 110$ \\
\hline \multicolumn{4}{|c|}{ 10.Orthostatic hypertension during tilt } \\
\hline Results & Grading & Value & Definition* \\
\hline Normal & & 0 & SBP $\leq 120 \%$ of baseline \\
\hline Abnormal & & 1 & SBP $>120 \%$ of baseline \\
\hline
\end{tabular}

Cerebral Blood Flow

\begin{tabular}{l|l|l|l|}
\hline 11.Valsalva maneuver & cerebral blood flow & \multicolumn{2}{l}{} \\
\hline Results & Grading & Value & Definition \\
\hline Normal & & 0 & $\geq 85 \%$ of baseline \\
\hline & Mild & 1 & $\geq 80$ and $<85 \%$ of baseline \\
\hline \multirow{3}{*}{ Abnormal } & Moderate & 2 & $\geq 65$ and $<80 \%$ of baseline \\
\cline { 2 - 4 } & Severe & 3 & $<65 \%$ of baseline \\
\hline
\end{tabular}

Using systolic CBFV. * Systolic CBFV at the end of phase 2, baseline is defined as the CBFV just before the Valsalva maneuver

12.Cerebral autoregulation

\begin{tabular}{|c|c|c|c|}
\hline Results & Grading & Value & Definition \\
\hline Normal & & 0 & \\
\hline Abnormal & & 1 & $\begin{array}{l}\text { Systolic CBFV > } 160 \mathrm{~cm} / \mathrm{s} \text { during phase } 4 \text { and (Systolic CBFV-Diastolic CBFV)phase } 4 \text { / (Systolic CBFV } \\
\text { - Diastolic CBFV)baseline ) }>140 \%\end{array}$ \\
\hline \multicolumn{4}{|c|}{ 13.Supine cerebral blood flow low } \\
\hline Results & Grading & Value & Definition \\
\hline Normal & & 0 & \\
\hline Abnormal & & 1 & $<$ lower normal limit \\
\hline \multicolumn{4}{|c|}{ 14.Supine cerebral blood flow high } \\
\hline Results & Grading & Value & Definition \\
\hline Normal & & 0 & \\
\hline Abnormal & & 1 & > upper normal limit \\
\hline \multicolumn{4}{|c|}{ 15.Cerebral blood flow response to tilt } \\
\hline Results & Grading & Value & Definition* \\
\hline Normal & & 0 & \\
\hline \multirow{3}{*}{ Abnormal } & Mild & 1,2 & \\
\hline & Moderate & 3- 4 & \\
\hline & Severe & $>4$ & \\
\hline
\end{tabular}

Using mean CBFV. CBFV should be measured at baseline, and at 1,5 and 10 minutes of tilt . ${ }^{*}$ At each measurement, assign the value 0 for normal results. Abnormal results are defined as CBFV less than normal limit. Normal limit is defined as follows: At 1.minute: normal drop of CBFV $\geq 90 \%$. Assign 1 for CBFV $80-89 \%$ of baseline, 2 for $70-79 \%$ of baseline and 3 for $<70 \%$ of baseline. AT 5 . minute: normal drop of CBFV $\geq 89 \%$. Assign 1 for CBFV $79-88 \%$ of baseline, 2 for $69-78 \%$ of baseline and 3 for $<69 \%$ of baseline. At 10 . minute: normal drop of CBFV $\geq 86 \%$. Assign 1 for CBFV $77-85 \%$ of baseline, 2 for $67-76 \%$ of baseline and 3 for $<67 \%$ of baseline. Total score is obtained by sum of all scores. For tilt longer than 10 minutes, additional measurements should be obtained every 5 minutes with the same grading as used at 10 th minute.

\section{Small Fiber Neuropathy}

\begin{tabular}{|c|c|c|c|}
\hline \multicolumn{4}{|c|}{ Sudomotor } \\
\hline \multicolumn{4}{|c|}{ 16.QSART } \\
\hline Results & Grading & Value & Definition \\
\hline Normal & & 0 & \\
\hline \multirow{3}{*}{ Abnormal } & Mild & 1 & $\begin{array}{l}\text { Single site } \geq 50 \% \text { and }<100 \% \text { or } \\
\text { Length-dependent pattern* or } \\
\text { Persistent sweat activity }\end{array}$ \\
\hline & Moderate & 2 & $\begin{array}{l}\text { Single site }<50 \% \text { or } \\
\text { Two or more sites }<100 \% \text { and } \geq 50 \%\end{array}$ \\
\hline & Severe & 3 & Two or more sites $<50 \%$ \\
\hline
\end{tabular}

${ }^{*} 100 \%=$ normal limit. At least 3 sites should be studied. *Length dependent pattern is defined as a reduction of the distal sweat volume more than $1 / 3$ of proximal value. 17.Sweat gland nerve fiber density

\begin{tabular}{|l|l|l|l|}
\hline Results & Grading & Value & Definition \\
\hline Normal & & 0 & \\
\hline
\end{tabular}


Citation: Novak P (2015) Quantitative Scale for Grading of Cardiovascular Autonomic Reflex Tests and Small Fibers from Skin Biopsies (QASAT). J Neurol Disord 3: 226. doi:10.4172/2329-6895.1000226

Page 5 of 11

\begin{tabular}{|c|c|c|c|}
\hline \multirow{4}{*}{ Abnormal } & Mild & 1 & Single site $<100 \%$ and $\geq 85 \%$ of lower limit \\
\hline & Moderate & 2 & $\begin{array}{l}\text { Single site }<85 \% \text { and } \geq 65 \% \text { or } \\
\text { two sites }<100 \% \text { and } \geq 85 \%\end{array}$ \\
\hline & Severe & 3 & $\begin{array}{l}\text { One site }<65 \% \text { or } \\
\text { two or more sites }<85 \% \text { and } \geq 65 \%\end{array}$ \\
\hline & Marked & 4 & $\begin{array}{l}\text { One site }<50 \% \text { or } \\
\text { two or more sites }<65 \%\end{array}$ \\
\hline \multicolumn{4}{|c|}{$\begin{array}{l}* 100 \%=\text { normal limit. At least } 1 \text { biopsy should be done at the distal leg }(10 \mathrm{~cm} \text { above the lateral malleolus), with a second site at the thigh ( } 20 \mathrm{~cm} \text { distal to the iliac } \\
\text { spine). }\end{array}$} \\
\hline \multicolumn{4}{|c|}{ Sensory } \\
\hline \multicolumn{4}{|c|}{ 18.Epidermal nerve fiber density } \\
\hline Results & Grading & Value & Definition \\
\hline Normal & & 0 & \\
\hline \multirow{4}{*}{ Abnormal } & Mild & 1 & Single site $<100 \% *$ and $\geq 70 \%$ \\
\hline & Moderate & 2 & $\begin{array}{l}\text { Single site }<70 \% \text { and } \geq 40 \% \text { or } \\
\text { two sites }<100 \% \text { and } \geq 70 \%\end{array}$ \\
\hline & Severe & 3 & $\begin{array}{l}\text { One site }<40 \% \text { or } \\
\text { two or more sites }<70 \% \text { and } \geq 40 \%\end{array}$ \\
\hline & Marked & 4 & $\begin{array}{l}\text { One site }<20 \% \text { or } \\
\text { two or more sites }<40 \%\end{array}$ \\
\hline \multicolumn{4}{|c|}{$\begin{array}{l}* 100 \%=\text { normal limit. At least } 1 \text { biopsy should be done at the distal leg }(10 \mathrm{~cm} \text { above the lateral malleolus), with a second site at the thigh ( } 20 \mathrm{~cm} \text { distal to the iliac } \\
\text { spine). }\end{array}$} \\
\hline \multicolumn{4}{|c|}{$\begin{array}{ll}19 . T o t a l \\
\text { 19core (items } 1-18)\end{array}$} \\
\hline Results & Grading & Value & Definition \\
\hline Normal & & 0 & \\
\hline \multirow{3}{*}{ Abnormal } & Mild & $1-5^{*}$ & \\
\hline & Moderate & $6-12^{*}$ & \\
\hline & Severe & $>12^{*}$ & \\
\hline
\end{tabular}

Table 1: Definition of QASAT

\begin{tabular}{|l|l|c|c|}
\hline 1. item & 2. item & R & P \\
\hline Sensory & Sudomotor Sweat gland & 0.36 \\
\hline Sensory & Sudomotor-QSART & 0.16 \\
\hline Sensory & Heart rate variability & 0.2 & 0.0001 \\
\hline Sudomotor -QSART & Sudomotor-Sweat glands & 0.16 & 0.001 \\
\hline Sudomotor-QSART & Heart rate variability & 0.21 \\
\hline Orthostatic hypotension & Blood flow response to tilt test & 0.32 \\
\hline R=Pearson's correlation coefficient & & 0.0001 \\
\hline
\end{tabular}

Table 2: Pairwise Correlations of QASAT sections.

The QASAT sections are grouped in 3 main categories: Cardiovascular (sections 1-10), Cerebral blood flow (sections 11-15) and Small fiber neuropathy (sections 16-18). The QASAT sections are defined as follows:

1) Heart Rate. The heart rate section assesses the presence of supine bradycardia, tachycardia, heart responses to tilt and heart rate variability. The latter uses the standard deep breathing test [35]. CASS uses the deep breathing test as a marker of cardiovagal or parasympathetic functions $[10,17]$. However, multiple factors affect the breathing tests including the sensory afferent pathways, parasympathetic and sympathetic modulation, chest mechanic, resting heart rate, volemic and cardiac status, therefore the non-leading term "heart rate variability" was adopted.

2) Blood Pressure. This section assesses the resting blood pressure, response of the blood pressure to the Valsalva maneuver and to tilt. In CASS, both the Valsalva maneuver and orthostatic hypotension were used to construct a sympathetic/adrenergic marker. In QASAT the association of blood pressure changes with a particular autonomic domain is avoided since the tilt provokes complex responses and blood pressure changes induced by tilt test depends upon integrative effect of neural (both sympathetic and parasympathetic), humoral and cardiovascular activity. The QASAT grades both reduced (orthostatic hypotension) and increased (orthostatic hypertension [48]) responses to the tilt.

3) Cerebral Blood Flow. This section assesses blood flow velocity from the middle cerebral artery. It uses both absolute values obtained in supine position and its response to Valsalva maneuver and tilt. Valsalva maneuver is also used to create an autoregulatory index (Table 1 , section 12).

4) Sudomotor. This section has two subsections, the functional which uses the QSART with grading taken from CASS. The second subsection is morphological which uses the sweat gland nerve fiber density.

5) Sensory. This section uses the epidermal nerve fiber density.

QASAT was validated using training and a testing dataset as follows. Initially, data from 100 participants ( 25 healthy controls, and patients 


\begin{tabular}{|c|c|c|}
\hline QASAT & CASS & Specificity/Sensitivity (\%/\%) \\
\hline Heart rate variability score $=0$ & Cardiovagal score $=0$ & $100 / 100$ \\
\hline Heart rate variability score $=1$ & Cardiovagal score=1 & $100 / 90.5$ \\
\hline Heart rate variability score $=2$ & Cardiovagal score $=2$ & $32.3 / 96.7$ \\
\hline Heart rate variability score $=3$ & Cardiovagal score $=3$ & $72.8 / 95.9$ \\
\hline Valsalva maneuver score $=0$ & Adrenergic score $=0$ & $100 / 83.4$ \\
\hline Valsalva maneuver score=1 & Adrenergic score $=1$ & $44.0 / 91.3$ \\
\hline Valsalva maneuver score $=2$ & Adrenergic score $=2$ & $64.9 / 78.8$ \\
\hline Valsalva maneuver score $=3$ & Adrenergic score $=3,4^{*}$ & $9.8 / 100$ \\
\hline $\mathrm{OH}$ score $=0$ & Adrenergic score $=0$ & $92.3 / 38.8$ \\
\hline OH score =1,2 & Adrenergic score $=1$ & $15.7 / 100$ \\
\hline $\mathrm{OH}$ score $=3,4$ & Adrenergic score $=2$ & $5.3 / 100$ \\
\hline OH score $=5,6$ & Adrenergic score $=3$ & $12 / 98$ \\
\hline OH score $>6$ & Adrenergic score $=4$ & $38.3 / 92.2$ \\
\hline $\mathrm{OH}+\mathrm{VM}$ Score $_{\mathrm{QASAT}}=0$ & Adrenergic score $=0$ & $96.0 / 93.1$ \\
\hline $\mathrm{OH}+\mathrm{VM}$ Score $_{\text {QASAT }}=1,2$ & Adrenergic score $=1$ & $43.0 / 71.5$ \\
\hline $\mathrm{OH}+\mathrm{VM}$ Score ${ }_{\text {QASAT }}=3-4$ & Adrenergic score $=2$ & $71.8 / 69.7$ \\
\hline $\mathrm{OH}+\mathrm{VM}$ Score ${ }_{\mathrm{QASAT}}=5-9$ & Adrenergic score $=3$ & $65.4 / 73.2$ \\
\hline $\mathrm{OH}+\mathrm{VM}$ Score $_{\text {QASAT }}>9$ & Adrenergic score $=4$ & $75.6 / 72.6$ \\
\hline CBS-tilt score $=0$ & Adrenergic score $=0$ & $45.9 / 61.5$ \\
\hline CBS-tilt score $=1,2$ & Adrenergic score $=1$ & $25 / 100$ \\
\hline CBS-tilt score $=3,4$ & Adrenergic score $=2$ & $19.5 / 100$ \\
\hline CBS-tilt score=>4 & Adrenergic score $=3$ & $61.4 / 46.3$ \\
\hline Sensory score $=0$ & Sudomotor-score $=0$ & $63.4 / 39.3$ \\
\hline Sensory score=1 & Sudomotor-score=1 & $15.1 / 82.3$ \\
\hline Sensory score=2 & Sudomotor-score=2 & $11.3 / 92.9$ \\
\hline Sensory score=3 & Sudomotor-score $=3$ & $7.8 / 95.4$ \\
\hline $\begin{array}{l}\text { Sudomotor sweat gland } \\
\text { score }=0\end{array}$ & Sudomotor-score $=0$ & $68.8 / 38.0$ \\
\hline $\begin{array}{l}\text { Sudomotor sweat gland } \\
\text { score }=1\end{array}$ & Sudomotor-score=1 & $10.8 / 91.8$ \\
\hline $\begin{array}{l}\text { Sudomotor sweat gland } \\
\text { score =2 }\end{array}$ & Sudomotor-score $=2$ & $8.33 / 91.4$ \\
\hline $\begin{array}{l}\text { Sudomotor sweat gland } \\
\text { score }=3\end{array}$ & Sudomotor-score $=3$ & $13.6 / 91.0$ \\
\hline \multicolumn{3}{|c|}{$\begin{array}{l}{ }^{*} \text { CASS-adrenergic grades } 3 \text { and } 4 \text { (severe and marked abnormalities) were combined } \\
\text { since the Valsalva maneuver score }{ }_{\text {OASAT }} \text { divides abnormalities into } 3 \text { grades only } \\
\text { (mild-moderate-severe) whereas CASS-adrenergic has } 4 \text { (mild-moderate-severe- } \\
\text { marked), CBS=cerebral blood flow score, VM=Valsalva maneuver, OH= orthostatic } \\
\text { hypotension. }\end{array}$} \\
\hline
\end{tabular}

Table 3: Sensitivities and specificities of QASAT.

with mild $(\mathrm{n}=25)$, moderate $(\mathrm{n}=25)$, and severe $(\mathrm{n}=25)$ autonomic failure according to CASS criteria were used to define criteria for grading abnormal results in each domain. Genetic algorithms [49] that are method for solving optimization problems mimicking process of natural selection were used for QASAT generation. The sections (heart rate, blood pressure, blood flow velocity, sudomotor and sensory) were determined using physiological and clinical judgment. Then initial QASAT criteria for degree of abnormalities (e.g. mild-moderatesevere-(market)) were generated randomly, which were then adjusted by successive iterations until the best match with CASS was achieved in differentiation of both the diagnostic groups and CASS severity. Once the best match was achieved, e.g. if the solution converged, the obtained criteria were used without changes in the remaining subjects. These patients were different from those used for the initial fine-tuning of the QASAT. QASAT was written in Matlab programming language (MathWorks, Natick, MA), and all scores were generated automatically.

CASS scores were also calculated automatically using Low's original description [35] that was modified to resolve several ambiguities [36].

Statistical analyses
QASAT was compared with CASS. Since the naming is different in QASAT compared to CASS, CASS-based cardiovagal score was compared with heart rate variability score (item 5, Table 1). The QASAT-based adrenergic failure score was defined as combination of orthostatic hypotension (item 7, Table 1) and Valsalva maneuver score (item 8, Table 1). Then CASS-based adrenergic failure score was compared with the QASAT-based adrenergic failure score as defined above. Total CBF score was defined as summation of all CBF sections.

One-way analysis of variance (ANOVA) was used to test the overall difference between normal, mild, moderate, severe and marked (for adrenergic failure only) CASS scores. The comparison was done for total QASAT scores as well for each section (for example QASAT- sensory, QASAT - sweat gland, etc.) If ANOVA showed overall significance, post hoc Tuckey test was used for mean comparisons of each scores. ANOVA was also used to analyze the overall difference between diagnostic groups. The comparison was done for total QASAT scores as well for each section. If they differed significantly, post hoc Tuckey test was used for mean comparisons.

Multiple linear regressions with covariates (age, sex, BMI, diagnoses) and Pearson's correlation coefficients $(r)$ between continuous variables were calculated. CASS was used as a gold standard for calculation of sensitivities and specificities. All statistical analysis used JMP 10.0 (SAS Institute, Inc., Cary, NC).

\section{Results}

The testing was completed in 719 patients. Patients were excluded from the study if they were taking medications that could affect the autonomic testing $(\mathrm{n}=43)$; were unable to complete the testing protocol $(n=4)$; had ECG or blood pressure signals of substandard quality due motion artifacts $(n=5)$; were on anticoagulation medication and therefore ineligible for biopsy for safety reasons $(n=8)$; lacked temporal bone window $(n=29)$; had severe anemia which affect cerebral blood flow $(n=12)$; had intracranial stenosis of the middle cerebral artery $(n=4)$; or declined skin biopsy $(n=2)$.

Data from 612 patients were included in the analysis. The following diagnostic groups were represented: diabetes mellitus (n,age $\pm \mathrm{sd}, \mathrm{f} / \mathrm{m}$ ) $(92,61.6 \pm 12.6,42 / 50)$, Parkinson disease $(88,70.9 \pm 10.8,36 / 52)$ and multiple system atrophy $(23,63.8 \pm 11.4,10 / 13)$. The remaining patients $(n=409)$ represent a heterogenous group with history of other disorders (typically with $\mathrm{n}<15$ ) than mentioned above; these disorders include migraine, hypertension, multiple sclerosis, atypical parkinsonism, coronary artery disease, unexplained dizziness, chronic fatigue syndrome and others.

The correlation between total QASAT and total CASS was $r=0.84$ $(\mathrm{p}<0.0001)$. ANOVA showed overall significance $(\mathrm{p}<0.0001)$ among graded autonomic failure implicating that the 4 grades of autonomic failure (none, mild, moderate, severe) according to the QASAT were significantly different. Posthoc comparisons showed a significant difference $(p<0.05)$ between each mean of graded autonomic failure (Figure 1A).

Five percent of patients that had normal total CASS score $(=0)$ but abnormal total QASAT score $(>0)$. ANOVA showed overall significance among diagnoses $(\mathrm{p}<0.0001)$ using both QASAT and CASS (Figure 1B). Posthoc pairwise comparisons showed a significant difference $(\mathrm{p}<0.05)$ between multiple system atrophy and Parkinson disease groups, but failed to distinguish Parkinson disease and diabetes groups for both CASS $(\mathrm{p}<0.94)$ and QASAT $(\mathrm{p}<0.136)$.

The correlation between cardiovagal score obtained from CASS 
Citation: Novak P (2015) Quantitative Scale for Grading of Cardiovascular Autonomic Reflex Tests and Small Fibers from Skin Biopsies (QASAT). J Neurol Disord 3: 226. doi:10.4172/2329-6895.1000226

Page 7 of 11

and heart rate variability score from QASAT was $r=0.93(\mathrm{p}<0.0001)$. The correlation between adrenergic failure score obtained from CASS and combined orthostatic hypotension and Valsalva maneuver score obtained from QASAT (e.g. adrenergic failure) was $0.82(\mathrm{p}<0.0001)$.
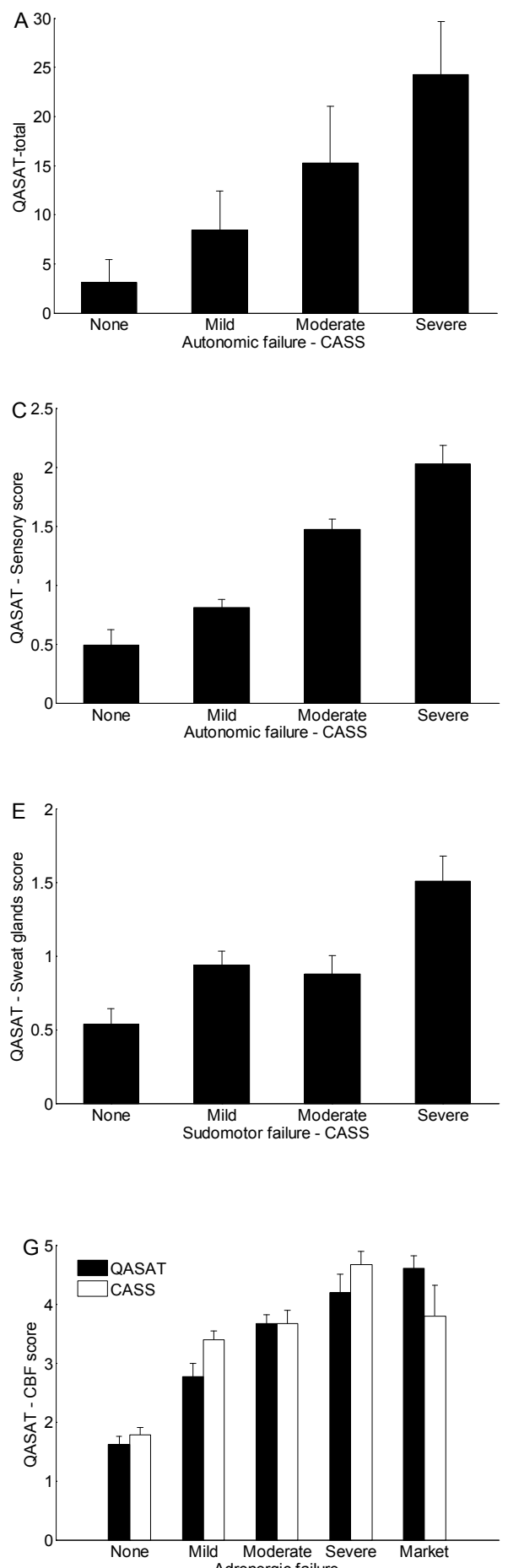

The selected sensitivities/specificities for each grade of abnormalities are in Table 3.

The correlation between CBF - total score and the adrenergic failure score from CASS was $r=0.29(\mathrm{p}<0.0001)$. The correlation coefficient
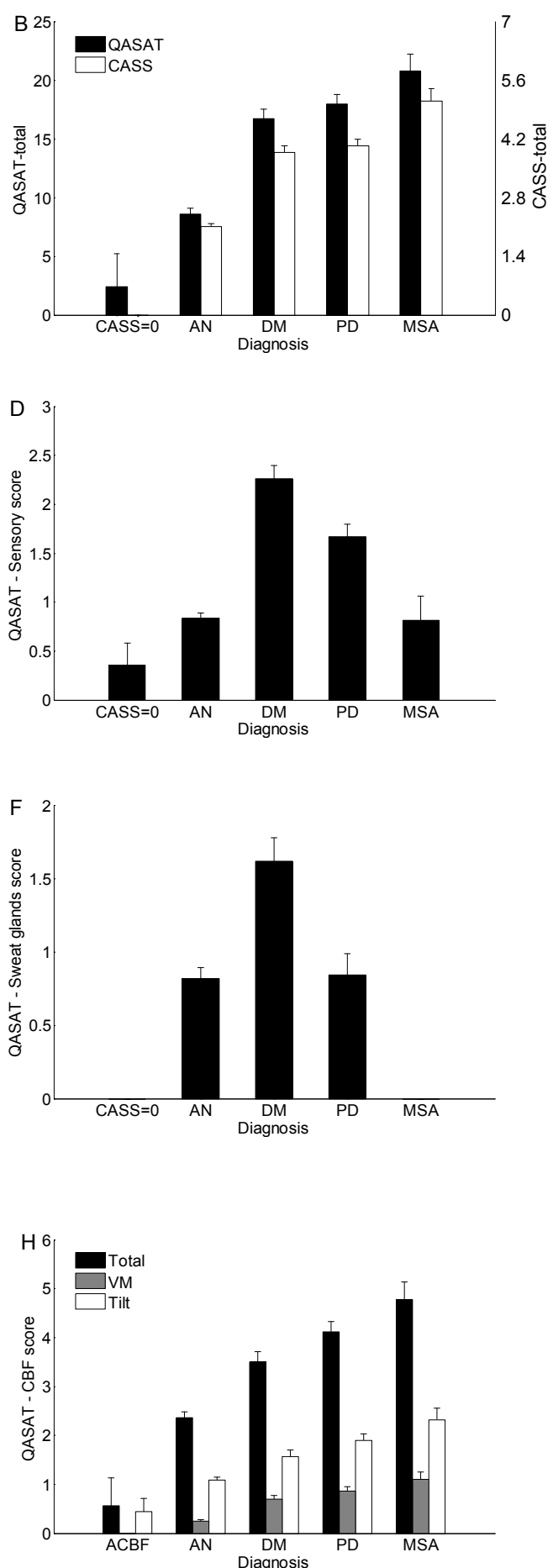

Figure 1: Grading of abnormalities using QASAT. In all panels (A-H), the bar graphs show means and respective standard errors except in panel A which shows standard deviations. In all panels ANOVA showed overall significance of differences among compared variables. Panels $\mathrm{G}$ and $\mathrm{H}$ show cerebral blood flow (CBF) scores. Panel G shows CBF scores at graded adrenergic failure obtained from CASS and QASAT. Note that CBF score proportionally increases with QASATadrenergic score but declines at marked adrenergic failure as defined in CASS. ACBF=abnormal CBF only with normal (equal to zero) all other CASS domains. "Total" on panel $\mathrm{H}$ indicates combined CBF score obtained by summation of all CBF sections, QASAT-based adrenergic failure score was defined as combination of orthostatic hypotension and Valsalva maneuver score.VM=Valsalva maneuver - CBF score, Tilt=Blood flow response to tilt score. 
between CBF-blood flow response to tilt score and the combined orthostatic hypotension and Valsalva maneuver score obtained from QASAT (e.g. adrenergic failure) was $r=0.32$ (p<0.0001).

ANOVA showed overall significance among graded adrenergic failure when stratified using either CASS or QASAT $(\mathrm{p}<0.0001$, Figure $1 \mathrm{G})$. ANOVA showed overall significance among diagnoses (Figure $1 \mathrm{H}$ ) for total CBF score $(\mathrm{p}<0.001)$. CBF subscores using tilt only $(\mathrm{p}<0.0004)$ or Valsalva maneuver $(\mathrm{p}<0.04)$ only were also overall significant among diagnosis groups $(\mathrm{p}<0.01$, Figure $1 \mathrm{H})$. The sensitivity and specificity of CBF score to detect abnormal CASS-adrenergic score was $61.5 \%$ and $46.3 \%$, respectively.

The sensory score was abnormal, e.g. consistent with small fiber neuropathy affecting sensory fibers, in $46.6 \%$ of patients. ANOVA of sensory score showed overall significance among graded autonomic failure from CASS as well as diagnoses ( $<<0.0001$, Figure $1 \mathrm{C}$ and $1 \mathrm{D}$ ). Posthoc pairwise comparisons showed significant difference between all groups $(\mathrm{p}<0.05)$. The sensitivity and specificity of sensory score to detect abnormal QSART was $39.3 \%$ and $63.4 \%$, respectively. The sensitivities and specificities stratified on degree of sudomotor failure are at Table 2.

The sudomotor score using sweat gland fiber density (sweat gland score) was abnormal, e.g consistent with small fiber neuropathy affecting sudomotor fibers, in $37.1 \%$ of patients. ANOVA of sweat gland score showed overall significance among graded sudomotor failure from CASS $(\mathrm{p}<0.0071)$ as well as diagnoses $(\mathrm{p}<0.00185$, Figure $1 \mathrm{E}$ and $1 F$ ). For sudomotor failure, posthoc pairwise comparison showed significant difference $(\mathrm{p}<0.05)$ only between severe failure and normal group. For diagnoses, posthoc pairwise comparisons showed significant difference between Parkinson disease, diabetes and MSA groups only $(\mathrm{p}<0.05)$. The sensitivity and specificity of sweat gland score to detect abnormal QSART was $38 \%$ and $68.4 \%$, respectively. The sensitivities and specificities stratified on degree of sudomotor failure are at Table 3. Correlations between selected QASAT sections are at Table 2 .

No overall significance at $\mathrm{p}<0.05$ level (Figure 2) among graded autonomic failure using either CASS (total, parasympathetic or adrenergic) or QASAT (total or sections) or diagnoses was revealed by ANOVA for baroreflex gain, initial response to the fast and slow tilt, LF/HF (both absolute and normalized and their ratios), Plasma catecholamines and indices of cerebral autoregulation. The LF/HF and plasma catecholamines were evaluated in 110 subjects only. Afterwards these tests were discontinued as a part of routine autonomic testing because the ANOVA was not significant.

\section{Discussion}

This report defines QASAT, an instrument for scoring the cardiovascular reflex and related small fiber tests. The QASAT was validated by comparing with CASS. QASAT differentiates diagnostic categories similar to CASS but scores wider range of abnormalities. A novel feature of QASAT is that it recognizes abnormalities below the normal range (e.g. supine low blood pressure, bradycardia, orthostatic hypotension) as well as above the normal range (e.g. supine/orthostatic hypertension, tachycardia). By that way both parasympathetic/ sympathetic failure and parasympathetic/sympathetic overactivity can be detected and graded. However, cardiovascular reflex tests depend upon complex mechanisms that are not restricted to autonomic nervous system. Therefore the nomenclature has been simplified reflecting the fact that QASAT grades the tests and does not impose or restrict interpretation of the results. For example, the leading term "cardiovagal domain" that is used for the deep breathing test in CASS has been replaced by non-leading term "heart rate variability". Furthermore, cardiovascular tests are enhanced by grading cerebrovascular responses during tilt and Valsalva maneuver.

QASAT is objective, fully quantitative and enables automatic calculation of all scores. QASAT uses clinically validated tests only with age and gender adjusted normative data where applicable. The scale is modular, each test is graded separately and grading does not require completion of all tests. Therefore, each laboratory can select their combination of tests. The individual tests can be combined based on clinical diagnostic needs, e.g. cerebral blood flow monitoring during tilt test can be useful in the evaluation of cerebral syncope. QASAT has an expanded dynamic range which allows to refine the results (total score range is $0-49$, while CASS score range is $0-10$ ).

Heart rate score captures wider spectrum of abnormalities than CASS-based cardiovagal domain including bradycardia, tachycardia and heart rate response to tilt. Detecting these abnormalities can aid in the diagnosis of parasympathetic overactivation, anxiety, hypovolemia, deconditioning, or postural orthostatic tachycardia syndrome. At the same time, heart rate variability score -that is a subset of heart rate score - is compatible with the parasympathetic cardiovagal domain score as defined in CASS.

The combination of the Valsalva maneuver-blood pressure and orthostatic hypotension scores is compatible with the CASS-based adrenergic domain that assesses severity of adrenergic failure. However QASAT uses relative changes in blood pressure whereas CASS uses absolute values. This difference affects sensitivity to detect adrenergic failure in patients with low baseline supine blood pressure. In these patients, CASS requires a larger drop in blood pressure than does QASAT to satisfy the criteria for orthostatic hypotension. QASAT also captures wider spectrum of abnormalities compared to CASS such as supine hyper- and hypotension and orthostatic hypertension. These abnormalities can reflect sympathetic failure or sympathetic overactivity. The naming follows historical tradition. Supine hypertension is defined as an absolute blood pressure at supine position while orthostatic hypertension is defined as an increase blood pressure provoked by standing.

The cerebral blood flow score (either total or blood flow response to tilt) correlates with the orthostatic blood pressure score and CASSbased adrenergic failure confirming that orthostatic hypotension is associated with orthostatic drop of cerebral blood flow. However, the correlation even it is significant, remains low, indicating that the blood pressure responses to tilt test is not an ideal proxy for the cerebral perfusion during the tilt that confirms previous findings [44]. Pressureflow relationship is nonlinear [50] and it is governed by the cerebral autoregulation that include multiple pathways [43-44]. Then it is no surprise that the genetic algorithms failed to converge for cerebral autoregulation to any meaningful clinical or physiological scoring criteria for all approaches described in methods since these methods assume linear pressure-flow relationship. Therefore a simple empirical index of autoregulation was incorporated into the QASAT that detects abnormal vasomotor reactivity induced by Valsalva maneuver. The main limitation of the transcranial Doppler is the lack of temporal bone window transparent for ultrasound in some patients. Furthermore, the normative values are machine dependent, and therefore each lab should develop its own references.

This study provides strong evidence that sensory and autonomic abnormalities coexist. Both sensory and sweat gland fiber densities 

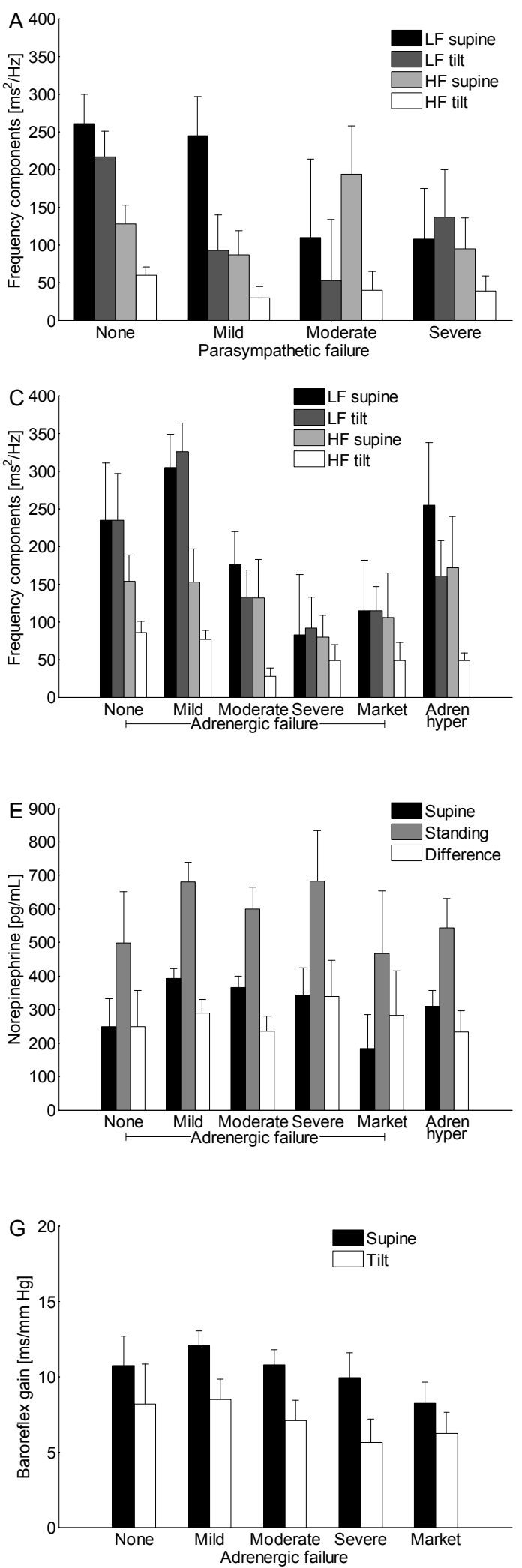
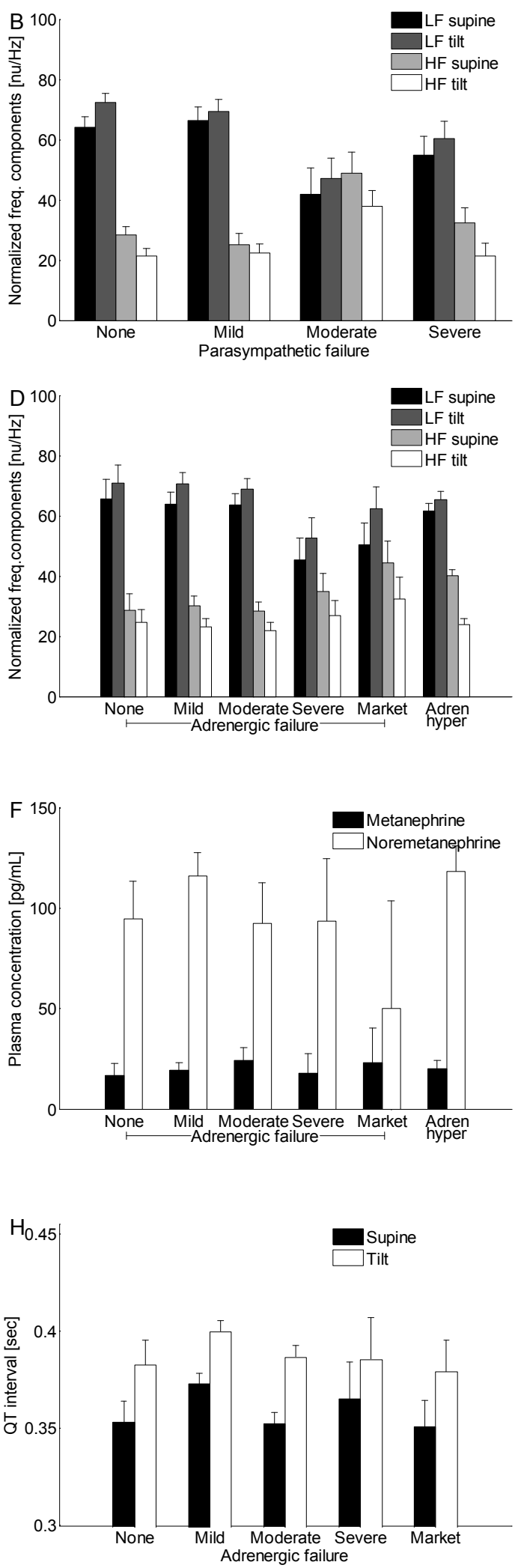

Figure 2: Failed tests. ANOVA did not show overall significant difference for all tests shown here. Bar graphs show means and respective standard errors. Graded parasympathetic and adrenergic failure shown in this figure was derived from QASAT. Adren hyper = Adrenergic hyperactivity score. 
Citation: Novak P (2015) Quantitative Scale for Grading of Cardiovascular Autonomic Reflex Tests and Small Fibers from Skin Biopsies (QASAT). J Neurol Disord 3: 226. doi:10.4172/2329-6895.1000226

Page 10 of 11

were reduced in small fiber neuropathies. The reduction of both types of fibers was disease dependent, being highest in diabetes followed by Parkinson disease. Furthermore, the fibers reduction was proportional to the severity of autonomic failure. Therefore inclusion of epidermal sensory and sweat gland fiber density evaluation enables more detailed grading of the severity of small fiber neuropathies and also helps to localize the lesion.

Low correlation of QSART with either epidermal or sweat gland fibers calls for caution when interpreting the QSART results. QSART might overestimate the sudomotor deficit [51] and retest reliability of the QSART is also limited [52-53].This study suggests that sweat gland fiber density might be used as an alternative to QSART.

\section{Negative Results}

Initial response to both slow and fast tilt, baroreflex gain, QT interval, plasma catecholamines and frequency analysis of the RR interval failed to segregate patients based on diagnostic categories, with or without dysautonomia and also the detected abnormalities were not proportional to the graded adrenergic, parasympathetic failure or generalized autonomic failure. Therefore these tests are unlikely to contribute significantly to routine autonomic or small fiber neuropathy testing. Nevertheless, these tests may have a value when used in special circumstances.

\section{Study Limitations}

The main limitation of the QASAT is that it remains to be validated as an objective monitoring tool of progression of symptoms and to measure benefits from a given therapy.

In conclusion QASAT is a validated, objective, fully quantitative instrument for grading of cardiovascular reflex tests, dysautonomia, associated small fiber neuropathies and cerebral blood flow.

\section{Acknowledgements}

The author thanks Dr. Lan Qin, Shane Stanek, Steve Smajkiewicz, Donald Chin and Lina Garcia for help in data collection.

The study was funded by the Department of Neurology, University of Massachusetts Medical School.

\section{References}

1. Ganzeboom KS, Mairuhu G, Reitsma JB, Linzer M, Wieling W, et al. (2006) Lifetime cumulative incidence of syncope in the general population: a study of 549 Dutch subjects aged 35-60 years. J Cardiovasc Electrophysiol 17: 11721176.

2. Chen LY, Shen WK, Mahoney DW, Jacobsen SJ, Rodeheffer RJ (2006) Prevalence of syncope in a population aged more than 45 years. Am J Med 119: 1088

3. Kenny RA, Bhangu J, King-Kallimanis BL (2013) Epidemiology of syncope/ collapse in younger and older Western patient populations. Prog Cardiovasc Dis 55: $357-363$.

4. Grossman SA, Chiu D, Lipsitz L, Mottley JL, Shapiro NI (2014) Can elderly patients without risk factors be discharged home when presenting to the emergency department with syncope? Arch Gerontol Geriatr 58: 110-114.

5. Soteriades ES, Evans JC, Larson MG, Chen MH, Chen L, et al. (2002) Incidence and prognosis of syncope. N Engl J Med 347: 878-885.

6. Linzer M, Yang EH, Estes NA 3rd, Wang P, Vorperian VR, et al. (1997) Diagnosing syncope. Part 1: Value of history, physical examination, and electrocardiography. Clinical Efficacy Assessment Project of the American College of Physicians. Ann Intern Med 126: 989-996.

7. Sloane PD, Coeytaux RR, Beck RS, Dallara J (2001) Dizziness: state of the science. Ann Intern Med 134: 823-832.

8. Radtke A, Lempert T, von Brevern M, Feldmann M, Lezius F, et al. (2011) Prevalence and complications of orthostatic dizziness in the general population. Clin Auton Res 21: 161-168.
9. Maarsingh OR, Dros J, Schellevis FG, van Weert HC, van der Windt DA, et al. (2010) Causes of persistent dizziness in elderly patients in primary care. Ann Fam Med 8: 196-205.

10. Low PA (2008) Laboratory evaluation of autonomic failure In PA Low, ed Clinical Autonomic disorders, Kluver-Lippincott, Philadelphia, USA.

11. Hilz MJ, Dütsch M (2006) Quantitative studies of autonomic function. Muscle Nerve 33: 6-20.

12. Freeman R, Chapleau MW (2013) Testing the autonomic nervous system. Handb Clin Neurol 115: 115-136.

13. Wieling W, Van Lieshout JJ, Ten Harkel AD (1998) Dynamics of circulatory adjustments to head-up tilt and tilt-back in healthy and sympathetically denervated subjects. Clin Sci (Lond) 94: 347-352.

14. Chandler MP, Mathias CJ (2002) Haemodynamic responses during head-up tilt and tilt reversal in two groups with chronic autonomic failure: pure autonomic failure and multiple system atrophy. J Neurol 249: 542-548.

15. Smith JJ, Porth CM, Erickson M (1994) Hemodynamic response to the upright posture. J Clin Pharmacol 34: 375-386.

16. Novak $P$ (2011) Assessment of sympathetic index from the Valsalva maneuver Neurology 76: 2010-2016.

17. Freeman R (2006) Assessment of cardiovascular autonomic function. Clin Neurophysiol 117: 716-730.

18. Callaghan BC, Cheng HT, Stables CL, Smith AL, Feldman EL (2012) Diabetic neuropathy: clinical manifestations and current treatments. Lancet Neurol 11: 521-534.

19. Vinik AI, Ziegler D (2007) Diabetic cardiovascular autonomic neuropathy. Circulation 115: 387-397.

20. Ziegler D, Zentai CP, Perz S, Rathmann W, Haastert B, et al. (2008) Prediction of mortality using measures of cardiac autonomic dysfunction in the diabetic and nondiabetic population: the MONICA/KORA Augsburg Cohort Study Diabetes Care 31: 556-561.

21. Kuehl M, Stevens MJ (2012) Cardiovascular autonomic neuropathies as complications of diabetes mellitus. Nat Rev Endocrinol 8: 405-416.

22. Cersosimo MG, Benarroch EE (2012) Autonomic involvement in Parkinson's disease: pathology, pathophysiology, clinical features and possible peripheral biomarkers. J Neurol Sci 313: 57-63.

23. Asahina M, Vichayanrat E, Low DA, lodice V, Mathias CJ (2013) Autonomic dysfunction in parkinsonian disorders: assessment and pathophysiology. J Neurol Neurosurg Psychiatry 84: 674-680.

24. Hoitsma E, Reulen JP, de Baets M, Drent M, Spaans F, et al. (2004) Small fiber neuropathy: a common and important clinical disorder. J Neurol Sci 227: $119-130$.

25. Lauria G, Lombardi R (2012) Small fiber neuropathy: is skin biopsy the holy grail? Curr Diab Rep 12: 384-392.

26. Lauria G, Lombardi R, Camozzi F, Devigili G (2009) Skin biopsy for the diagnosis of peripheral neuropathy. Histopathology 54: 273-285.

27. Hoeijmakers JG, Faber CG, Lauria G, Merkies IS, Waxman SG (2012) Smallfibre neuropathies--advances in diagnosis, pathophysiology and management Nat Rev Neurol 8: 369-379.

28. Devigili G, Tugnoli V, Penza P, Camozzi F, Lombardi R, et al. (2008) The diagnostic criteria for small fibre neuropathy: from symptoms to neuropathology. Brain 131: 1912-1925.

29. Ensrud KE, Nevitt MC, Yunis C, Hulley SB, Grimm RH, et al. (1992) Postura hypotension and postural dizziness in elderly women. The study of osteoporotic fractures. The Study of Osteoporotic Fractures Research Group. Arch Intern Med 152: 1058-1064.

30. Rutan GH, Hermanson B, Bild DE, Kittner SJ, LaBaw F, et al. (1992) Orthostatic hypotension in older adults. The Cardiovascular Health Study. CHS Collaborative Research Group. Hypertension 19: 508-519.

31. Mader SL, Josephson KR, Rubenstein LZ (1987) Low prevalence of postural hypotension among community-dwelling elderly. JAMA 258: 1511-1514.

32. Tinetti ME, Speechley M, Ginter SF (1988) Risk factors for falls among elderly persons living in the community. N Engl J Med 319: 1701-1707. 
Citation: Novak P (2015) Quantitative Scale for Grading of Cardiovascular Autonomic Reflex Tests and Small Fibers from Skin Biopsies (QASAT). J Neurol Disord 3: 226. doi:10.4172/2329-6895.1000226

Page 11 of 11

33. Liu BA, Topper AK, Reeves RA, Gryfe C, Maki BE (1995) Falls among older people: relationship to medication use and orthostatic hypotension. J Am Geriatr Soc 43: 1141-1145.

34. Ooi WL, Hossain M, Lipsitz LA (2000) The association between orthostatic hypotension and recurrent falls in nursing home residents. Am J Med 108: $106-$ 111

35. Low PA (1993) Composite autonomic scoring scale for laboratory quantification of generalized autonomic failure. Mayo Clin Proc 68: 748-752.

36. Novak P (2011) Quantitative autonomic testing, Journal of Visual Experimentation, 53.

37. Rickards CA, Newman DG (2003) A comparative assessment of two techniques for investigating initial cardiovascular reflexes under acute orthostatic stress. Eur J Appl Physiol 90: 449-457.

38. Bernardi L, De Barbieri G, Rosengård-Bärlund M, Mäkinen VP, Porta C et al. (2010) New method to measure and improve consistency of baroreflex sensitivity values. Clin Auton Res 20: 353-361.

39. Spallone V, Ziegler D, Freeman R, Bernardi L, Fontoni S, et al. (2011) Cardiovascular autonomic neuropathy in diabetes: clinical impact, assessment, diagnosis, and management. Diabetes Metab Res Rev 27: 639-653.

40. Task Force of the European Society of Cardiology and the North American Society of Pacing and Electrophysiology: Heart rate variability: standards of measurement, physiological interpretation and clinical use (1996) Circulation 93: $1043-1065$.

41. Garland EM, Hooper WB, Robertson D (2013) Pure autonomic failure. Handb Clin Neurol 117: 243-257

42. Goldstein DS, Holmes C, Sharabi Y, Brentzel S, Eisenhofer G (2003) Plasma levels of catechols and metanephrines in neurogenic orthostatic hypotension. Neurology 60: 1327-1332.
43. Franco Folino A (2007) Cerebral autoregulation and syncope. Prog Cardiovasc Dis 50: $49-80$

44. Novak V, Novak P, Spies JM, Low PA (1998) Autoregulation of cerebral blood flow in orthostatic hypotension. Stroke 29: 104-111.

45. Zhang R, Zuckerman JH, Giller CA, Levine BD (1998) Transfer function analysis of dynamic cerebral autoregulation in humans. Am J Physiol 274: H233-241.

46. Tiecks FP, Douville C, Byrd S, Lam AM, Newell DW (1996) Evaluation of impaired cerebral autoregulation by the Valsalva maneuver. Stroke 27: 11771182.

47. Tiecks FP, Lam AM, Aaslid R, Newell DW (1995) Comparison of static and dynamic cerebral autoregulation measurements. Stroke 26: 1014-1019.

48. Fessel J, Robertson D (2006) Orthostatic hypertension: when pressor reflexes overcompensate. Nat Clin Pract Nephrol 2: 424-431.

49. Forrest S (1993) Genetic algorithms: principles of natural selection applied to computation. Science 261: 872-878.

50. Hu K, Lo MT, Peng CK, Liu Y, Novak V (2012) A nonlinear dynamic approach reveals a long-term stroke effect on cerebral blood flow regulation at multiple time scales. PLoS Comput Biol 8: e1002601.

51. Sletten DM, Weigand SD, Low PA (2010) Relationship of Q-sweat to quantitative sudomotor axon reflex test (QSART) volumes. Muscle Nerve 41: 240-246.

52. Peltier A, Smith AG, Russell JW, Sheikh K, Bixby B, et al. (2009) Reliability of quantitative sudomotor axon reflex testing and quantitative sensory testing in neuropathy of impaired glucose regulation. Muscle Nerve 39: 529-535.

53. Berger MJ, Kimpinski K (2013) Test-retest reliability of quantitative sudomotor axon reflex testing. J Clin Neurophysiol 30: 308-312. 